\title{
Un demi-siècle d'historiographie religieuse du Canada français à travers les congrès de la S.C.H.E.C.
}

\section{Pierre Savard}

Volume 50, numéro 1, 1983

Bilan de l'histoire religieuse au Canada

Canadian Catholic History: A survey

URI : https://id.erudit.org/iderudit/1007034ar

DOI : https://doi.org/10.7202/1007034ar

Aller au sommaire du numéro

Éditeur(s)

Les Éditions Historia Ecclesiæ Catholicæ Canadensis Inc.

ISSN

0318-6172 (imprimé)

1927-7067 (numérique)

Découvrir la revue

Citer cet article

Savard, P. (1983). Un demi-siècle d'historiographie religieuse du Canada

français à travers les congrès de la S.C.H.E.C. Sessions d'étude - Société

canadienne d'histoire de l'Église catholique, 50(1), 47-58.

https://doi.org/10.7202/1007034ar

Tous droits réservés @ Les Éditions Historia Ecclesiæ Catholicæ Canadensis Inc., 1983
Ce document est protégé par la loi sur le droit d'auteur. L'utilisation des services d'Érudit (y compris la reproduction) est assujettie à sa politique d'utilisation que vous pouvez consulter en ligne. 


\title{
Un demi-siècle d'historiographie religieuse
}

\author{
du Canada français \\ à travers les congrès de la S.C.H.E.C.
}

Pour rendre justice à l'oeuvre de la Société qui s'étale sur un demi-siècle, il convient de rappeler brièvement ses origines et son évolution ainsi que le milieu tant historiographique que socio-religieux dans lequel elle a évolué. Il n'est point question ici de raconter dans le détail l'histoire interne de la Société. Plutôt que de relater les péripéties d'un organisme soutenu à coup de bonne volonté pendant un demi-siècle et ce, avec une fidélité remarquable - histoire du reste non sans intérêt par ce qu'elle nous apprend sur les hommes et les femmes qui l'ont faite et sur le climat dans lequel ils ont travaillé - nous avons choisi de nous attacher à l'aspect le plus visible de la Société, à savoir sa production historique publiée dans les actes de ses congrès annuels appelés aussi Sessions d'étude ou, autrefois, Rapport annuel.

Dans un article publié il y a à peine dix ans, le père Gaston Carrière, historien et témoin privilégié puisqu'il compte parmi les plus prolifiques des collaborateurs aux congrès annuels, a dit l'essentiel sur les origines et le développement de la Société. Née de l'idée de James F. Kenney, archiviste aux Archives publiques du Canada, la Société prit à l'origine comme modèle l'American Catholic Historical Association, fondée en 1919, florissante société qui publiait la Catholic Historical Review. Au début du siècle, la France, pour sa part, avait vu les chercheurs en histoire ecclésiastique se regrouper en une Société d'histoire de l'Église de France qui publiait elle aussi un périodique depuis 1910. C'est l'Allemagne qui avait ouvert la voie avec la fameuse société Görres aux séries de publications scientifiques renommées dès les années 1880. À Louvain, la Revue d'histoire ecclésiastique s'était affirmée dès 1900 la grande revue internationale d'histoire du catholicisme et fournissait par sa bibliographie un instrument indispensable aux chercheurs en histoire religieuse et même aux historiens curieux d'autres secteurs de l'historiographie. En somme, en 1933, les Canadas français et anglais ne manquaient pas de modeles. 
La Société, dont l'objet est le passé de toute l'Église catholique du Canada, regroupera, dans la pensée de ses fondateurs, francophones et anglophones en deux sections divisées selon la langue. Les congrès annuels sont conjoints jusqu'en 1963. Depuis cette date, les réunions ont lieu séparément. La Société semble s'être rendue à une évidence que les habitués de congrès dits nationaux de ce pays connaissent bien. $\mathrm{Vu}$ le faible taux réel de bilinguisme et la divergence des intérêts, anglophones et francophones se lassent tôt ou tard de se réunir. De plus, l'espace étudié par la section française est autre que celui de l'anglaise. Mis à part les missions du Grand Nord et les poches de peuplement dans l'Ouest, les Canadiens français sont avant tout intéressés par le monde religieux francophone qui s'étend de Moncton à Sudbury quand ce n'est pas celui du Québec tout court. Les Canadiens anglais, par contre, ne peuvent se désintéresser d'un catholicisme qui pousse des racines de Terre-Neuve à la Colombie britannique. D'où l'éclatement inévitable du congrès annuel.

La publication des actes en un seul volume et des liens organiques au niveau des deux exécutifs constituent donc les seuls signes tangibles de l'unité de la Société.

Encouragée par les évêques et en particulier par le cardinal Villeneuve, la Société connaît un départ lent puis se développe pour atteindre au milieu des années 1940 près de cinq cents membres dans sa seule section française, ce qui représente un sommet inégalé depuis. Dès les origines, la Société est ouverte aux laïcs: elle a compté 11 laïcs sur 25 présidents pendant les quarante premières années. Cependant, des 283 communications publiées durant la même période, 83 seulement l'ont été par des laïcs: le nombre élevé des clercs s'explique du fait que certains ecclésiastiques se révèlent bien prolifiques: 5 prêtres donnent de 5 à 8 communications chacun des débuts à 1973. La proportion des laïcs croît de façon très significative après 1960 comme on le verra plus loin. L'activité de la Société englobe tout le Canada français et non seulement les grands centres: de 1933 à 1983, elle a tenu son congrès annuel dans 25 diocèses dont 10 hors Québec. Québec a reçu la Société 6 fois, Montréal et Ottawa 4 fois chacun.

Les buts de la Société, tels que définis par les fondateurs, sont ambitieux. Elle veut "grouper les étudiants" c'est-à-dire les chercheurs et les amateurs de l'histoire catholique du Canada. Elle veut les aider à transmettre au public le fruit de leurs recherches: d'où la publication du rapport annuel. Elle vise aussi à créer des instruments de travail (catalogues d'archives, collections de documents, etc.). Cet objectif 
sera loin d'être atteint. Il sera repris en partie par le Rapport de l'archiviste de la Province de Québec, puis après 1970, avec plus de succès et plus de suite, par le Centre de recherche en histoire religieuse de l'Université Saint-Paul. Cependant, le père Sheehan de la section anglaise a lancé en 1965 une bibliographie couvrant toute l'histoire religieuse canadienne. Continuée en 1970 par le père Hanrahan puis par le père Hogan en 1976, cet outil fort bien fait est vite devenu indispensable à tous les chercheurs. Recensant livres et périodiques, il complète les bibliographies courantes de la Canadian Historical Review et de la Revue d'histoire de l'Amérique française. Quant aux buts originels d'établir des dépôts de documents et d'objets historiques, il sont restés lettre morte. Les progrès des institutions d'archives depuis une vingtaine d'années ont répondu à ce voeu. Enfin, la Société visait à élever le niveau de l'enseignement de l'histoire dans les maisons d'éducation catholiques. La forte proportion de professeurs de collèges classiques dans la Société dès le début peut laisser croire qu'elle a exercé alors une bonne action dans ce domaine.

Ainsi, laissant à d'autres les diverses fonctions d'inventaire d'archives ou de didactique qu'elle avait rêvé de remplir, la Société a consacré l'essentiel de ses énergies à l'organisation de congrès annuels et à la publication des actes. De plus, à partir de 1965 comme nous l'avons précisé plus haut, elle publie une précieuse bibliographie courante annexée aux actes des congrès annuels.

***

C'est à ses fruits qu'on juge l'arbre. Le grand oeuvre de la Société étant la tenue des congrès annuels et la publication des actes, nous nous pencherons sur ces productions pour en dégager quelques caractères. Nous avons procédé d'une façon un peu sommaire, comparant la production de deux décennies. La première est celle des années 1941 à 1950 alors que la Société, fondée depuis sept ans, a, après quelques tâtonnements, atteint sa vitesse de croisière. La seconde va de 1971 à 1980 : c'est à peu près celle qu'on connaît encore aujourd'hui. Nous limitons nos remarques aux textes publiés qui représentent près de neuf dixièmes des textes présentés aux congrès. Ce qui permet de dire que celui qui est invité à parler au congrès est presque automatiquement assuré de la publication. Les textes non publiés semblent être soit des textes non rédigés ou des communications que les auteurs veulent retravailler ou encore des textes promis ailleurs.

Nous avons retenu d'examiner ici le statut (clerc ou laïc; séculier ou régulier), la distribution spatiale des sujets d'étude, les "genres" et 
secteurs de l'histoire abordés (biographie, institutions, etc.) et enfin les périodes étudiées.

Un premier trait saute aux yeux: la proportion clercs/laïcs se modifie beaucoup entre 1940 et 1980 . Dans la décennie 1940, on compte deux clercs pour un laïc parmi les conférenciers publiés; dans la décennie 1970-1980, les deux groupes sont à égalité.

Historiens clercs et laïcs se distinguent quant à la production. Les premiers ont tendance à s'intéresser plus à l'histoire interne de l'Église. Souvent gardiens d'archives ou ayant un accès facile aux archives diocésaines ou à celles de leur congrégation, ils disposent d'un matériau riche et inexploité. Documentation qui peut remonter à des époques reculées comme celle du Séminaire de Québec ou porter sur des régions immenses comme celle des Oblats. Les travaux qu'ils présentent reflètent les faiblesses et les forces de leur documentation.

Ces historiens clercs se limitent trop souvent aux horizons de leur paroisse ou de leur diocèse. Quand ils sont d'une congrégation, ils affichent une tendance nette (ou est-ce simplement leur mandat d'historiographe de l'ordre?) à se limiter à éclairer ou à célébrer les gloires de leur famille religieuse. On songe irrésistiblement ici au mot de l'abbé Henri Bremond: "le jésuite se lève tôt chaque matin pour écrire la vie édifiante d'un autre jésuiste." Sinon à cet autre mot d'ecclésiastique: "Vanter son curé, quelle pitié; vanter son vicaire, quelle misère!" Ainsi, sur la vingtaine de communications des années 1940 produites par des religieux et religieuses, seize portent sur la congrégation de leur auteur. Cependant, cette tendance s'atténue avec le temps. Dans les années 1970, moins de la moitié de la vingtaine de communications présentées par des religieux et religieuses traitent de la congrégation de leur auteur. Soulignons en passant que la production abondante sur les communautés religieuses aide à comprendre la bonne place des religieuses parmi les auteurs et les sujets traités et donne ainsi aux femmes tant auteurs que sujets une place respectable dans les actes des congrès annuels.

Les historiens laïcs se montrent intéressés à l'histoire de l'Église catholique moins en elle-même que comme élément de la société canadienne-française. Dans les années 1940, les rapports entre l'Église et l'État et la situation légale de l'Église constituent des thèmes récurrents. Plus nombreux et mieux outillés que leurs prédecesseurs, des historiens laïcs des années 1970 ouvrent des chantiers neufs. Le plus souvent, ce sont de jeunes diplômés des départements d'histoire qui 
produisent en quantité de bon ouvriers de l'histoire depuis les années 1960.

Le caractère ambulant des congrès souvent tenus dans les diocèses périphériques aide à diversifier régionalement sujets d'études et collaborateurs. Dans les années 1940, si le tiers des articles porte sur l'histoire générale du catholicisme canadien-français, les deux tiers traitent spécifiquement de questions liées à une région. On y trouve une belle diversité qui reflète en bonne partie les lieux des congrès. Saint-Hyacinthe et la vallée du Richelieu, la région de Nicolet et le diocèse de Joliette comptent parmi les plus étudiées avec le Manitoba. Viennent ensuite, loin derrière, les régions de Montréal, Québec et Sherbrooke.

Trente ans plus tard on trouve le même souci de diversification spatiale. Des 61 communications publiées, 38 évoquent l'histoire dans le cadre diocésain ou régional. Chicoutimi et le Lac-Saint-Jean apparaissent le plus souvent ( 7 articles) suivis de Gaspé, Saint-Hyacinthe et Sherbrooke ( 5 et 4 ). Québec et Ottawa suivent avec 3 articles chacun. Le Manitoba compte deux articles (après avoir connu un congrès mémorable et une pluie d'articles à la fin de la décennie précédente).

Les exposés portent donc le plus souvent sur l'espace diocésain dans lequel se déroule le congrès. Mais cette règle souffre heureusement moult exceptions, qui enlèvent de l'homogénéité aux actes mais élèvent souvent le niveau de la publication. Ces communications portent soit sur un problème plus vaste ou sur une autre région.

Tant dans les années 1940 que dans les années 1970, c'est la biographie qui reste le genre le plus souvent pratiqué. Trois laïcs méritent l'attention d'un exposé dans les années 1940 contre une douzaine de clercs incluant trois évêques. Dans les années 1970 on n'entend plus parler des laïcs tandis que cinq des sept biographies ont trait à des évêques.

L'organisation ecclésiastique reçoit aussi une attention priviiégiée: plus de 12 exposés dans les années 1940 qui s'expliquent, il est vrai, par un congrès autour du thème de la paroisse. Dans les années 1970, on trouve encore 7 communications sur les questions de juridiction et de relations ecclésiastiques internes. Et nous n'incluons pas ici les exposés sur le problème voisin des rapports de l'Église 
et de l'État qui compte 7 exposés dans les années 1940 et 3 dans les années 1970.

L'éducation, et plus particulièrement l'oeuvre des collèges classiques, constitue un autre domaine de choix: 5 exposés durant les années 1940 et 9 pour les années 1970.

L'histoire des congrégations religieuses est cultivée tout au long de ces deux périodes. Au moins 6 exposés ont trait aux congrégations dans les années 1940 dont 4 aux congrégations féminines. Le totai, 9 dans les années 1970, est à peu près partagé également entre congrégations masculines et féminines. Ces chiffres doivent être majorés du fait que l'action de congrégations a pu être classée dans un sousthème comme celui des missions.

Les articles sur le sentiment religieux, la spiritualité et la pastorale sont relativement peu nombreux : 5 au total pour chaque période étudiée. Rappelons cependant qu'on trouve de riches notations dans les biographies et monographies de congrégations citées plus haut. L'histoire de l'Église reste celle des clercs tandis que «le peuple chrétien» sert de décor assez passif et mal connu.

On est surpris de voir des secteurs tout à fait sous-développés: l'action catholique, la catéchèse, l'action sociale et le syndicalisme, la colonisation, les rapports entre l'Église et la vie économique, les relations ou tout au moins la coexistence avec les autres confessions et les relations inter-ethniques dans l'Église catholique. Tous ces riches thèmes n'ont fait l'objet que d'un exposé chacun.

Malgré trois communications dans les années 1940 sur les missions au Canada et trois autres dans les années 1970 sur les Prêtres des Missions-Étrangères, on reste surpris de peu de place accordée à ce secteur si important dans le passé de l'Église catholique canadienne. Il est vrai que le domaine reste encore à peu près inexploré en dehors de quelques travaux de propagande missionnaire.

L'histoire de l'art religieux n'est représentée que par deux exposés dans les années 1940 et le même nombre dans les années 1970. C'est peu compte tenu de la richesse du matériau et de son apport à l'intelligence du religieux.

Des domaines capitaux pour la compréhension du monde actuel autant que du passé sont encore terra incognita dans les actes comme, par exemple, la vie morale et les organisations de jeunesse. 
La répartition des articles suivant l'époque étudiée offre un équilibre remarquable et beaucoup de stabilité. Dans les années 1940, le Régime français compte pour $16 \%$ des articles; dans les années 1970 , cette proportion est de $22 \%$. La période qui va de la Conquête à la Confédération compte pour $30 \%$ des articles dans les années 1940; elle baisse à $15 \%$ dans les années 1970 . La période après 1867 compte pour $36 \%$ des articles dans les années 1940. Dans la décennie 1970, nous distinguons deux sous-périodes. Les années de 1867 à 1929 et celles de 1929 à nos jours se partagent assez également les $60 \%$ des articles. Signalons que les articles non "périodisables» pendant les deux décennies constituent moins de $15 \%$ de chaque total.

En somme, on remarque une importance primordiale accordée à la période qui précède immédiatement la période contemporaine. Quant aux articles traitant de la période contemporaine, ils confinent le plus souvent au témoignage plus qu'ils ne constituent une étude historique, c'est-à-dire critique. Notons que le Régime français n'est pas surreprésenté comme dans la Revue d'histoire de l'Amérique française d'avant 1970. La place ample faite à l'histoire des cent dernières années vient sans doute du caractère itinérant des congrès qui se retrouvent souvent dans des diocèses jeunes ou tout le moins fondés après 1840 .

L'action de la Société est loin de se dérouler en vase clos. Aussi est-il utile de s'arrêter brièvement sur les conditions de l'Église catholique, de la société canadienne-française et de l'historiographie pendant le dernier demi-siècle pour mieux comprendre l'oeuvre de la Société.

La Société naît dans les années 1930 en un temps où quelques esprits inquiets s'interrogent déjà sur le catholicisme d'ici comme en témoignent la Relève ou la Revue dominicaine du temps. On est aussi au plus creux de la crise économique. L'action catholique connaît alors une belle expansion au Canada français avec, surtout, la Jeunesse ouvrière catholique et la Jeunesse étudiante catholique. Les missions étrangères sont elles aussi en pleine floraison. Puis vient la guerre et surtout l'après-guerre, période dont les craquements annoncent les années 1960. L'urbanisation s'accélère alors et la société de consommation s'étend. Après 1960 les digues éclatent: sécularisation de la pensée, dé-cléricalisation des institutions, chute brutale de la 
pratique religieuse et du recrutement clérical, désaffection à l'endroit du religieux puis, après 1970, engouement chez plusieurs pour les cultes et les sectes jusqu'ici sans contact avec la culture canadiennefrançaise.

Tous ces ébranlements et bien d'autres restent en général absents $\mathrm{du}$ questionnement historiographique. Même des phénomènes plus proprement religieux comme la surenchère d'orthodoxie des années 1950 ou la poussée mariale de la même époque suscitent relativement peu d'études historiques de nature critique. Tout se passe comme si la Société était pour beaucoup d'esprits le refuge où on célèbre les grandes heures et les grandes figures rassurantes d'un passé proche ou lointain, ou l'occasion de répondre à des questions que personne sauf un historien loin de son temps peut penser soulever. Disons cependant que des progrès dans les méthodes de l'historiographie se sont faits sentir à partir de 1960 surtout. De nombreux et jeunes collaborateurs tant laïcs que clercs ont diversifié le discours de la Société, le rendant plus critique et généralement moins laudatif.

À l'origine, la Société était le fait d'un petit nombre d'historiens surtout clercs où les autodidactes de l'histoire comme les Maheux et les Maurault dominaient. Aujourd'hui, la Société ouvre plus grands ses congrès à des conférenciers venant d'horizons plus larges: historiens de métier de toutes les générations et spécialistes des sciences humaines comme la sociologie.

Force est de constater cependant, et ce sans doute à cause du caractère régional des thèmes, que les amateurs aux intérêts étroitement locaux ou congréganistes occupent encore dans les congrès une place demesurée. Faut-il préciser qu'il n'est pas ici question de déprécier l'histoire régionale ni celle d'une congrégation particulière? Mais Clio a ses règles selon lesquelles on doit juger l'artisan de l'histoire à quelque échelle qu'il travaille. Un manque d'esprit critique appliqué à une documentation unilatérale portant sur des questions mal posées conduit à des résultats décevants quelle que soit l'envergure du sujet abordé.

En dépit d'un bilan quantitatif qui impressionne, la Société ne saurait réclamer le monopole de la production en histoire religieuse. En 1933, les rares historiens du religieux publiaient déjà depuis longtemps. Ils continueront de le faire dans le Bulletin des recherches historiques, dans les Mémoires de la Société royale du Canada sans 
parler des Cahiers des Dix ni des périodiques de congrégations comme Études oblates ou Lettres du Bas-Canada des jésuites. À partir de 1947, la Revue d'histoire de l'Amérique française accueille largement la prose des historiens du religieux. Après 1970, la mode de l'histoire des idéologies et de l'histoire sociale favorise la parution de nombreux articles dans Recherches sociographiques et de quelques-uns dans Histoire sociale/Social History. La prolifération des revues d'histoire régionale depuis les années 1960 comme les Cahiers d'histoire de la Gaspésie et qui publient beaucoup d'articles sur le passé religieux, aide aussi à comprendre l'abondance de la production. Rappelons enfin que la section anglaise de la Société publie à l'occasion dans les actes des articles de qualité sur l'Église au Canada français. On peut suivre ce mouvement dans les excellentes bibliographies courantes de la RHAF et dans celle de la Société. Ainsi, on constate que les auteurs d'histoire religieuse n'ont jamais manqué de débouchés. Et la production a été incontestablement stimulée par l'existence de la Société qui, au surplus, a fourni un forum national au sens canadien-français du terme, pour les historiens du catholicisme d'ici.

Des groupes d'historiens du catholicisme se sont également constitués parallèlement à la Société. Ainsi, le 4 octobre 1970 avait lieu à Saint-Gervais-de-Bellechasse le premier colloque du Groupe d'étude des religions populaires lancé par le dominicain Benoît Lacroix. Douze colloques annuels (sauf en 1979) ont suivi. Malgré l'objet quelque peu fuyant et imprécis du Groupe, les colloques ont donné lieu à des travaux historiques de valeur. Sans résoudre la question complexe à souhait de la religion populaire, à commencer par la notion même de religion populaire, les colloques ont fourni une belle démonstration de la fécondité de l'approche par thèmes en histoire religieuse. Le mieux réussi des colloque reste Les pèlerinages au Québec tenu à l'Université du Québec à Trois-Rivières en 1976 et publié sous ce titre aux Presses de l'Université Laval en 1981.

Dans les années 1970 a fleuri à Laval le Laboratoire d'histoire religieuse animé par le dynamique Hervé Gagné. Le Laboratoire a organisé des colloques et constitué un lieu d'échanges pour les historiens du religieux de Laval et d'ailleurs. Sous son égide est publiée une collection de travaux aux Presses de l'Université Laval. Le CELAT (Centre d'étude sur la langue, les arts et les traditions populaires des francophones en Amérique du Nord) a recueilli en partie l'héritage du Laboratoire. 
La Société ayant pour but de faire connaître le passé de l'Église catholique canadienne, on peut légitimement s'interroger sur son public et sur son rayonnement. La liste des membres de la section française de 1979 comprenait 138 personnes et une centaine d'institutions. Les personnes sont surtout des historiens tandis que les institutions sont avant tout des bibliothèques d'universités ou de congrégations religieuses. Les actes des congrès connaissent ainsi une bonne diffusion dans les bibliothèques. Un membre sur six de la Société est une femme ou une congrégation féminine. Une soixantaine d'abonnés se recrutent dans des provinces autres que le Québec soit surtout l'Ontario (Ottawa y occupant la bonne place), le Manitoba et le Nouveau-Brunswick. Une douzaine d'abonnements viennent des États-Unis. Les neuf autres sont surtout des bibliothèques parisiennes et romaines.

Les actes sont dépouillés dans de grands répertoires bibliographiques tel celui de la Revue d'histoire ecclésiastique de Louvain, le Religion Index Two: Multi-Author Works, la bibliographie de la Revue d'histoire de l'Amérique française, celle de la Canadian Historical Review, dans le Bulletin signalétique 527 (histoire et sciences de la religion), dans la Bibliographie annuelle de l'histoire de France et dans America, History and Life sans oublier la bibliographie de la S.C.H.E.C. mentionnée plus haut.

L'utilisation dans des travaux de synthèse et la citation dans des survols critiques constituent d'autres indices du rayonnement d'une publication. À ce chapitre, les actes ne font pas mauvaise figure. Dans le tome second de sa classique synthèse The Christian Church in Canada, John Sargent Moir cite à l'occasion les rapports annuels sur des questions qui n'ont pas été traitées ailleurs. Dans son étude des congrégations féminines au Canada français, soeur Marguerite Jean cite dix articles relatifs à son sujet: huit viennent des rapports annuels de la Société. Dans la bibliographie de leur analyse des communautés religieuses, Denault et Lévesque donnent quelque 25 titres d'articles: le rapport annuel y figure onze fois contre quatre pour la Revue d'histoire de l'Amérique française. Quant à Chaussé, dans la bibliographie copieuse de son Lartigue, il ne donne pas moins de 23 articles du rapport annuel ayant trait surtout au premier tiers du XIX siècle. Nous avons vu que la Société s'est toujours intéressée aux "francophones hors Québec». Le fait trouve confirmation dans l'état de la recherche sur l'Église catholique en Acadie par Fernand Arsenault dans la Revue de l'Université de Moncton de mars 1982. Des 14 articles retenus, six sont tirés des actes de la Société (dont l'un en anglais). 
La tâche des responsables de la Société est des plus délicates. Garder la faveur d'un large public de curieux de l'histoire religieuse suivant la pensée des fondateurs et éviter de sombrer dans la société pour initiés. Garder aussi le contact avec les petits et moyens centres sans pour cela tomber dans le "localisme» étriqué. Assurer un minimum de rigueur en matière de science historique tout en restant lisible à une époque où l'historiographie savante est tombée entre les mains de praticiens qui semblent n'écrire que pour être lus de quelques pairs. Et enfin, éviter le piège par excellence de ces sociétés, soit le panégyrique ou l'hagiographie.

\section{$* * *$}

Un cinquantenaire ne saurait se réduire à évoquer le travail accompli. L'histoire du catholicisme au Canada et au Canada français semble promis à un bel avenir. Certes le nombre des ouvriers de qualité reste paradoxalement peu considérable malgré la prolifération des diplômés en histoire depuis vingt ans et l'accroissement des vocations tardives d'historiens amateurs portés par le nostalgisme, le régionalisme et le culte du patrimoine qui nous hantent. Toutefois, des conditions nouvelles favorisent l'histoire religieuse. L'histoire dite sociale est devenue l'étendard des historiens qui se veulent vivants. L'histoire sociale a braqué ses projecteurs sur les travailleurs, tant dans leurs conditions de vie que dans leur organisation, les femmes, la vie urbaine et d'autres secteurs qui tôt ou tard rencontrent le phénomène religieux. Déjà les actes des congrès de la Société reflètent, timidement il est vrai, ce renouveau.

Une autre chance de l'histoire religieuse, c'est l'ouverture à une histoire plus soucieuse du vécu. Les ébranlements de la religion chez nous depuis deux décennies ont amené des penseurs, clercs surtout mais aussi laïcs, à s'interroger plus profondément sur l'essentiel du catholicisme. Ces inquiétudes n'ont pas été sans profiter aux historiens qui ne sont pas entièrement coupés de leur temps et qui, suivant le beau mot de Lucien Febvre, «interrogent les morts» certes mais «en fonction des vivants».

Mon ultime mot sera pour vous remercier de votre patience face à ces considérations parfois sans ménagement, considérations qui peuvent détonner au milieu de célébrations d'un demi-siècle de travail fait dans la joie et la collaboration. Croyez que mes propos restent inspirés par le désir de rendre meilleur ce forum stimulant et essentiel qui nous fait mieux connaitre l'homme d'ici dans une dimension essentielle de son 
vécu. Croyez aussi qu'ils sont inspirés par ma fidélité depuis près de vingt ans à suivre les travaux de notre Société. Et, considération non négligeable, de vingt ans et plus à goûter l'amitié de plusieurs d'entre vous, amitié qui nous réconforte dans cette longue traque souvent solitaire de l'homo religiosus d'ici.

Pierre SA V.ARD

Département d'histoire

Université d'Ottawa 